\title{
EL ENFOQUE CUALITATIVO EN LA INVESTIGACIÓN JURÍDICA, PROYECTO DE INVESTIGACIÓN CUALITATIVA Y SEMINARIO DE TESIS.
}

\author{
THE QUALITATIVE APPROACH IN LEGAL RESEARCH, QUALITATIVE \\ RESEARCH PROJECT AND THESIS SEMINAR
}

\author{
Medardo Nizama Valladolid ${ }^{1}$ \\ Universidad Nacional Mayor de San Marcos. \\ ORCID: 0000-0003-2804-7508 \\ mnizamav@unmsm.edu.pe \\ Luz María Nizama Chávez ${ }^{2}$ \\ Universidad de San Martín de Porres. \\ luznichav@gmail.com \\ Perú
}

\section{SUMARIO}

- Introducción

- Categorías en las que se afianza el enfoque cualitativo en la investigación jurídica

- Proyecto de Investigación Cualitativa

- Seminario de Tesis

- Conclusiones

- Recomendaciones

- Fuentes de Información

\section{RESUMEN}

En este trabajo se explica el desarrollo conceptual del enfoque cualitativo en la investigación jurídica, con el fin de despertar el ánimo e interés por su aplicación, en el ámbito de la investigación en el Posgrado de Derecho y Ciencia Política. Por eso, el estudio comprende el análisis de la relación o nexum existente entre el enfoque cualitativo y la investigación jurídica, así como su desenvolvimiento formal o conceptual: cognitivo, metodológico, político, etc.

En relación a los objetivos propuestos se refiere que, gracias al análisis conceptual, se ha logrado explicar el desarrollo en que se encuentra el enfoque cualitativo en la investigación jurídica en el Posgrado de Derecho y Ciencia Política. También se da cuenta que un primer resultado obtenido está en la apreciación de la relación o nexum existente entre el enfoque cualitativo y la investigación jurídica: dos categorías en estudio, las mismas que muestran una sustantividad y significación importante, basada en una fundamentación epistemológica, intencional, voluntaria e interpretativa. En general, los resultados obtenidos en el trabajo están en saber ahora, en nuestro medio, que la aplicación del enfoque cualitativo es inicial y con una clara tendencia a su crecimiento, conforme

\footnotetext{
${ }^{1}$ Bachiller, Abogado, Magíster, Doctor por la Universidad Nacional Mayor de San Marcos. Título de Profesor por la Universidad Nacional de Trujillo. Egresado de Maestría en Educación, Universidad Nacional Enrique Guzmán y Valle (La Cantuta), Docente Principal en Pre y Posgrado de Derecho, UNMSM. Profesor invitado en las Escuelas de Posgrado: Derecho de la UNSAAC, UNSCH (Ayacucho), UNHEVAL (Huánuco), UNS (Chimbote)

${ }^{2}$ Alumna de pregrado de la Facultad de Derecho de la Universidad de San Martín de Porres.
} 

tesis.

The qualitative approach in legal research, qualitative research project and thesis seminar

lo demuestran las conclusiones ideales o formales obtenidas en el presente estudio. Entre las dificultades encontradas tenemos la carencia de una literatura especializada, no solo de autores nacionales sino también de estudiosos extranjeros. Otra dificultad está en la falta de dominio cognitivo y técnico necesario para la construcción de estos trabajos con enfoque jurídico cualitativa.

\section{ABSTRACT}

In this brief work we explain the conceptual development of the qualitative approach in legal research in order to awake the spirit and interest in its application in the field of research in the postgraduate law and political science. Therefore, the study includes the analysis of the relationship or nexum between the qualitative approach and legal research as well as his formal or conceptual development: cognitive, methodological, political, etc.

In relation to the proposed objectives, we refer that thanks to the conceptual analysis, it has been possible to explain the development of the qualitative approach in legal research in the law and political science postgrade. We also realize that a first result obtained is in the assessment of the relationship or nexum between the qualitative approach and legal research the two categories in a study, which show an important substantivity and significance, based on an epistemological foundation, intentional, voluntary and interpretive. In general, the results obtained in the work are now knowing, in our environment, that the application of the qualitative approach is

\footnotetext{
${ }^{3}$ Que se puede explicar y entender a partir de la situación de crisis por la que atraviesa la globalización neoliberal, del declive del hegemonismo imperial y de la crisis económica, social y política que vive la sociedad norteamericana. En este marco general se pueden comprender las medidas antiinmigrantes del régimen de Trump, la propuesta de vuelta a casa de las empresas norteamericanas, el cuestionamiento
}

initial and with a clear tendency towards its growth, as evidenced by the ideal or formal conclusions of the present study.

Among the difficulties encountered, we lack the specialized literature not only of national authors but also of foreign scholars. Another difficulty is in the lack of cognitive and technical mastery necessary for the construction of these works with a qualitative legal approach.

\section{PALABRAS CLAVE}

Enfoque cualitativo; investigación jurídica; investigación cualitativa; seminario de tesis; estado del enfoque cualitativo; situaciones del enfoque cualitativo.

\section{KEYWORDS}

Qualitative approach; legal research, qualitative research; thesis seminar, state of qualitative approach; qualitative approach situations

\section{INTRODUCCIÓN}

Es esta una breve reflexión teórico conceptual en el contexto de la teoría formal de la racionalidad teórica, de un mundo globalizado ${ }^{3}$ actual. Entraña una finalidad didáctica porque muestra una visión analítica e ilustrativa, respecto a la relación o nexum entre el enfoque cualitativo y la investigación jurídica. Asimismo, es útil saber por nosotros mismos que el enfoque cualitativo sí es realmente un aplicativo en la tarea que tiene el jurista de resolver los problemas de la realidad socio jurídica; y, no como antes se pensaba que dicho

\footnotetext{
a los Tratados de Libre Comercio y las críticas al funcionamiento de la Organización del Tratado del Atlántico Norte (OTAN). Las medidas que viene aplicando no inciden en la solución de los serios problemas que tiene la sociedad norteamericana y algunas de estas servirán para reforzar el poder de los grupos dominantes de los Estados Unidos. (Raúl More UNC) Domenica 12-03-17
} 
enfoque solo era propio de las ciencias naturales. Ahora se sabe que este enfoque también resulta útil al estudiante de postgrado para abordar temas de actualidad, como la inseguridad jurídica, corrupción, cuestiones de género, etc.

Sin embargo, nos inquieta el resultado obtenido en otros trabajos y que consisten en el hallazgo de que dicho enfoque apenas es un aplicativo inicial, en los proyectos de investigación presentados en las maestrías y doctorado de posgrado de Derecho y Ciencia Política. Empero, alienta saber que dicho enfoque tiene al mismo tiempo una clara tendencia hacia su crecimiento; conforme a lo expresado por algunos destacados epistemólogos y filósofos de la ciencia; y esto alienta a la comunidad académica el surgimiento inicial oportuno de este enfoque cualitativo. Por lo demás, se da cuenta del interés y motivación que ha causado en los autores, el estudio y aplicación de este enfoque, el mismo que entraña una riqueza conceptual muy útil para todos los estudiantes en el referido posgrado.

En lo concerniente a las siguientes preguntas planteadas: ¿Cuál es el sentido y significación del desarrollo conceptual del enfoque cualitativo en la investigación jurídica en los estudiantes, egresados y profesores del Postgrado de Derecho y Ciencia Política? ¿Cuáles son los conceptos o categorías en los que se afianza el enfoque cualitativo en la investigación jurídica en los estudiantes y docentes del Posgrado de Derecho y Ciencia Política? ¿Cuáles son las técnicas cualitativas más utilizadas en la investigación jurídica en los estudiantes y docentes del Posgrado de Derecho y Ciencia Política? Al respecto, dichas preguntas han sido resueltas en el curso de la investigación.

Respecto de los objetivos trazados en el estudio, estos se han cumplido a cabalidad, en tal sentido se ha logrado explicar $y$ analizar el desarrollo conceptual o teórico del enfoque cualitativo en la investigación jurídica.

Por todo esto, el propósito fundamental de este trabajo es defender la conciencia y la mente del estudiante contra el irracionalismo de no crear riqueza ni mejorar la calidad de vida, no obstante, estar inmersos en la era o sociedad del conocimiento. Se espera sintonizar con las inquietudes de los profesores, estudiantes y egresados del posgrado y ser útiles para lograr que el estudio genere satisfacción, aliento y entusiasmo, que tanto necesitamos para efectuar la gestión y cultura de proyectos sobre enfoques epistemológicos, particularmente, el enfoque cualitativo.

\section{CATEGORÍAS EN LAS QUE SE AFIANZA EL ENFOQUE CUALITATIVO DE LA INVESTIGACIÓN JURÍDICA}

Una iniciativa personal del estudiante del Posgrado de Derecho y Ciencia Política señala que la futuridad de las naciones depende cada vez más del conocimiento; otra iniciativa manifiesta que el Perú y otras naciones del continente, muestran una desolación por la falta de ciencia, tecnología e investigación. Por estas iniciativas se ha preparado este trabajo para ceder la palabra a los cultores de los enfoques epistemológicos, particularmente el enfoque cualitativo.

Por más esfuerzos que han hecho los investigadores, hasta el presente no ha sido posible saber si la investigación ha sido capaz de progresar. Aunque no puede saberse con certeza --pese a la crisis-- si la investigación ha progresado significativamente en el ámbito de las 

tesis.

The qualitative approach in legal research, qualitative research project and thesis seminar

ciencias sociales ${ }^{4}$ y humanas; sin embargo, la gente percibe que en esta segunda década la investigación, ha crecido enormemente y ha causado un hondo impacto no solo en el pensamiento científico sino también en el pensamiento social y político. También se ha dicho que un estudiante de Posgrado de Derecho y Ciencia Política, que no conozca $^{5}$ hoy cuáles son los principales enfoques o vertientes de la investigación actual y desconozca su relación con los problemas científicos, sociales y políticos, está en desventaja para ayudar en la construcción de una nueva sociedad peruana, con ciudadanos grandes y grandes peruanos, sin sueños ni pesadillas.

Bernal, C. (2015, p.27-28) en su libro Fundamentos de la Investigaciòn, con estilo agradable, escribe un análisis reflexivo sobre la sociedad latinoamericana y la investigación. Un análisis optimista, vigoroso, reflexivo, y crítico; en que el citado autor aborda la crisis que tratan de superar los países subdesarrollados, que afecta profundamente a las instituciones públicas, particularmente, a las universidades públicas, que tienen a su cargo la investigación, la misma que constituye una función esencial $y$ obligatoria. Como tal, Bernal sabe dónde estriban las verdaderas causas de la competitividad y sabe también el momento en que la sociedad debe aprender los principios básicos del método científico y técnicas de investigación. Más aun, sabe que el estudio de las disciplinas y su enseñanza, es precario y pragmático.

\footnotetext{
${ }^{4}$ Las ciencias sociales se encuentran en un proceso de revisión y cuestionamiento de los fundamentos y propuestas teóricas, en particular del propio logos científico de la modernidad. Ver ponencia presentada en el XXVI Congreso de la Asociación Latinoamericana de Sociología, Guadalajara, México, agosto del 2007.
}

Específicamente, la esencia de la enseñanza es profesionalizante y sin relación alguna con la investigación.

Lo importante de Bernal es la manera cómo plantea, no solo el problema sino también la forma de solución que propone. Así afirma que para salir de la crisis y ocupar un lugar en el espectro de la sociedad del conocimiento, es necesario crear voluntad política y generar políticas públicas orientadas a la transformación humana $\mathrm{y}$ científica. De igual forma expone que es menester realizar cambios sustantivos en la administración pública; y luego juntos construir una nueva sociedad que valore la comunidad científica, como orientadora de la construcción de su propio destino. Es decir, una sociedad en la que los pueblos subdesarrollados asuman un rol activo en la preparación y ejecución de proyectos para explicar la vida social de los pueblos. De igual forma, Bernal afirma que el conocimiento generado por las disciplinas en los países desarrollados no resulta suficiente y adecuado, para utilizarse en cualquier otro país, sino que es necesario realizar investigación propia para cada contexto, a fin de lograr el desarrollo de los pueblos atrasados y afectados por la crisis arriba anotada.

\section{Categoría cognitiva y metodológica.}

Las ideas que se abordan en este punto son significativas e importantes para la formación espiritual y una cultura de investigación avanzada en el estudiante investigador. Nociones cognitivas que entrañan un breve análisis interpretativo,

5 En verdad, aquel estudiante universitario que no esté al tanto de la miseria científica y tecnológica que afecta al Perú y otros países de la región, no está a la altura de su tiempo. Ahora se piensa que los modelos teóricos y los métodos de las ciencias sociales ya no son los más apropiados y las interrogantes que se formulan ya no permiten reproducir niveles significativos de la realidad. 
pero antes de abordarlo conviene revisar algunas ideas de la filosofía de la ciencia. Con el estudio de tales ideas, y de acuerdo con Dynnik, et al, Historia de la Filosofía (1962, p.103-105) y Aristóteles Obras Completas (1947, T. II, p. 45 y 52), se percibe el significado de las siguientes reflexiones filosóficas y metodológicas.

Primero: Al hablar de la "ciencia enciclopédica" de Aristóteles, sus comentaristas identifican la amplitud universal de los intereses científicos del filósofo griego. Segundo: El estagirita no solo estudió los problemas de filosofía sino también los escollos de la lógica, psicología, ciencias naturales, historia, etc. Tercero: La crítica de Aristóteles a la filosofía de Platón ha desempeñado un papel importante en la historia del pensamiento filosófico. Igualmente, formuló graves objeciones contra el idealismo platónico. Cuarto: De la misma forma, Aristóteles demostraba siempre la infecundidad de la teoría platónica de las ideas al postular para cada cosa singular su propia "idea", con lo cual, en vez de explicar efectivamente los fenómenos de la naturaleza, no hacía más que duplicar el número de objetos que exigían explicación. Quinto: Entre tanto, la argumentación misma de Platón no es muy convincente, según señala con toda justicia Aristóteles, por cuanto, si se explica las cosas por medio de las "ideas", hay que admitir entonces que las "ideas" son las causas de las cosas; empero, el divino Platón niega que exista una razón causal entre las "ideas" y las cosas. Sexto: Por lo mismo, a Aristóteles le parecía imposible que la esencia de las cosas se hallara en un mundo trascendente, separada de las cosas mismas. Después de negar la existencia del reino de las "ideas" y de condenar el intento de Platón encaminado a descubrir la esencia de las cosas fuera de las cosas mismas (...). Por lo demás, Aristóteles "elabora la teoría de que la esencia (la sustancia) se encuentra en las cosas mismas. Aristóteles formula también una teoría de las cuatro causas: a)
La causa material o materia; b La causa formal o forma; c) La causa eficiente o agente, y d) La causa final o fin. Séptimo: Por último, la teoría del conocimiento de Aristóteles desempeñó un papel importante en la historia de la filosofía griega".

Él se apartaba radicalmente de las ciencias modernas, como la epistemología, entendida hoy -según Bunge -en su libro Epistemología, (1982, p. 21,22)- como una "ciencia propiamente dicha", que "se ocupa de problemas filosóficos que se presentan de hecho en el curso de la investigación científica o en la reflexión acerca de los problemas, métodos y teorías de la ciencia"; una epistemología que "propone soluciones claras a tales problemas, en particular, soluciones consistentes en teorías rigurosas e inteligibles, así como adecuadas a la realidad de la investigación científica, en lugar de teorías confusas o inadecuadas a la experiencia científica"; una epistemología que "es capaz de criticar programas y aún resultados erróneos, así como de sugerir nuevos enfoques promisorios". Una nueva epistemología -subraya Bunge (1982 p. 24)"que aborda los nuevos problemas "lógicos", "semánticos", "gnoseológicos", "metodológicos", "ontológicos", "axiológicos", "éticos" y "estéticos". Y, por último -concluye Bunge- la sociedad necesita "una epistemología viva en contacto estrecho con la investigación científica y útil a la filosofía en general como a la ciencia y, a través de esta, a la sociedad. No faltan los problemas ni las herramientas formales necesarias para abordarlos: falta solo la conciencia de que tales problemas existen y de que es preciso ocuparse de ellos en lugar de hacerlo con problemas bizantinos y aburridos".

Con las ideas que se abordan en este punto, el estudiante nuevamente percibe que necesita aún más fortalecer su acervo con ideas epistemológico- jurídicas actuales. A este respecto Aranzamendi en su libro Epistemología y la Investigación 

tesis.

The qualitative approach in legal research, qualitative research project and thesis seminar

Cualitativa y Cuantitativa en Derecho (2008, p. 45), explica que al investigador jurídico le interesa el estudio de la "epistemología jurídica, como sustento del conocimiento de la ciencia del Derecho y facilitan el estudio de los hechos o fenómenos de relevancia jurídica y social; así como también, conocer el significado, contenido, alcances, límites, métodos; $\mathrm{y}$, sobre todo, las orientaciones y expresiones de la cientificidad del derecho.

Por último, Aranzamendi (2008, p. 175) expresa: "Para sostener aún más la esencia cualitativa del derecho y que, en consecuencia, deberían tener presente los investigadores sobre los fenómenos jurídicos, son los fines del Derecho. El fin supremo del derecho es la justicia. Tiene como fin la constante búsqueda de la justicia. La justicia como sustrato del derecho es un valor cualitativo y es su esencia cualitativa lo que diferencia al derecho de las demás ciencias sociales, concluye el citado autor". Por su parte, Humberto Cerroni, citado por A. Espinoza (2008, p.73) considera que para sostener la misma esencia cualitativa debe tenerse en cuenta el objeto del derecho, el mismo que puede conducir a dos conclusiones: a) Construir las categorías jurídicas abstraídas de las relaciones sociales concretas y b) Construir categorías jurídicas que reproducen una estructura social determinada.

Por último, dentro de esta visión cognitivometodológica se necesita tener el dominio de la investigación interactiva (interaccionismo). Sobre este punto, decía Jacqueline Hurtado (2011, p. 105) que es ésta "una modalidad de investigación interactiva, pero fundamentalmente orientada a las ciencias sociales, $y$ dependiendo de la vertiente puede incorporar la participación de la comunidad estudiada. La investigación interactiva tiene aplicaciones no solo en el campo de las ciencias sociales, sino en cualquier otra disciplina".

\section{Investigación jurídica}

\subsection{Concepto}

Si observamos la realidad de la ciencia del derecho pronto surgirá una de las tantas interrogantes que fluyen de dicha realidad: ¿Existe investigación jurídica? El estudiante siempre se ha preguntado esto y la respuesta ha sido siempre: $\mathrm{Si}$ existe investigación jurídica; sin embargo, los hallazgos de la realidad jurídica no son espectaculares como los de medicina, física o biología y otras ciencias naturales. Los descubrimientos obtenidos en las disciplinas jurídicas, no se traducen en progresos tecnológicos, sino a la inversa, son disposiciones normativas con cierta motivación y relevancia social. Pues la experiencia muestra que, desgraciadamente con leyes ni códigos, no se resuelve aún la pobreza de la mitad de la población ni la enorme desigualdad existente en Lima y otras regiones del país. (Amat y León, El Perú nuestro de cada día, 2012)

Es así, que el autor Fix- Zamudio (1995, p. 416) sin pretender configurar un concepto de investigación jurídica nos recuerda que puede concebirse la investigación jurídica como "la actividad intelectual que pretende descubrir las soluciones jurídicas adecuadas para los problemas que plantea la vida social de nuestra época, cada vez más dinámica y cambiante, lo que implica también la necesidad de profundizar en el análisis de dichos problemas, con el objeto de adecuar el ordenamiento jurídico a dichas transformaciones sociales, aun cuando formalmente parezca anticuado"

\subsection{Objeto}

En su libro Metodología de la Investigación Jurídico Social, Solís Espinoza (2008, p. 47) refiere que "tradicionalmente el objeto central del estudio jurídico se concentra en el mundo normativo, de cuya base se 
derivan y fundamentan, directa $o$ indirectamente, los diversos desarrollos doctrinarios, históricos y filosófico jurídicos. Sin embargo, el objeto específico de conocimiento del Derecho puede ser comprendido también desde varias perspectivas, es así que desde su caracterización o su naturaleza óntica podemos vislumbrar estas tendencias".

"De este modo, -el citado Solís (2008, p. 48)- expresa que según el predominio o tendencia del ámbito de interés, su materia de estudio será fundamentalmente la realidad normativa con su abanico multiobjetal de vertientes (dogmáticajurídica, filosófico-jurídica, históricojurídica, teórico-jurídica, etc.); o bien su objeto de investigación será la realidad social empírica relacionada a la dimensión normativa (estudio socio-jurídico)".

Creemos que el precitado autor está en lo cierto, por cuanto, sostiene que los estudios jurídicos no solamente tienen una orientación normativista, sino que también pueden comprender otras perspectivas. Al menos es lo que observa en las escuelas y unidades de posgrado del país, una cierta coincidencia en la necesidad urgente de una transformación fundamental, no solo en el plano de los métodos de enseñanza sino también en el campo de la educación superior.

\subsection{Enfoques en la investigación jurídica}

El vocablo enfoque es tal en cuanto consiste en un palabra que el Diccionario de la Lengua Española (2001, p. 912) lo define como la "acción y efecto de enfocar" y, la voz enfocar, según el mismo Diccionario, quiere decir "dirigir la atención o el interés hacia un asunto o problema desde unos supuestos previos, para tratar de resolver dichos problemas".

\footnotetext{
${ }^{6}$ No solo los epistemólogos, sino también, otros investigadores asociaron el enfoque con ideas del
}

Por su parte, Carlos Sainz de Robles (1977, p. 436) en su Diccionario español de sinónimos y antónimos, el vocablo " enfoque" tiene el sentido de "orientar", "enfilar", "dirigir", "encaminar" y "examinar", etc.

Por lo demás, varios son los epistemólogos ${ }^{6}$ que asocian la idea de "enfoque" con la noción de "ruta", "dirección", "vertiente", "tendencia", "aproximación", etc.

Escribe Hernández Sampieri en su obra Metodología de la Investigación (2014, p. 4): "En el curso de la historia de la ciencia han surgido diversas corrientes de pensamiento (...) que han abierto diferentes rutas en la búsqueda del conocimiento. No es el caso revisar y profundizar por ahora en ellas; sin embargo, y debido a las diferentes premisas que la sustentan, desde el siglo pasado, tales corrientes se "polarizaron" en dos aproximaciones principales de la investigación: el enfoque cuantitativo y el enfoque cualitativo". "Ambos enfoques emplean procesos cuidadosos, metódicos y empíricos en su esfuerzo para generar conocimiento, por lo que la definición previa de investigación se aplica a las dos por igual. En términos generales - prosigueestos métodos utilizan cinco estrategias similares y relacionadas (Grinnell, 1997) entre si: $1^{\mathrm{a}}$, Si llevan a cabo, la observación y evaluación de fenómenos; $2^{\mathrm{a}}$, $\mathrm{Si}$ establecen suposiciones e ideas como consecuencia de la observación y evaluación realizadas; $3^{\mathrm{a}}$, Si demuestran el grado en que las suposiciones e ideas tienen fundamento; $4^{\mathrm{a}}, \quad \mathrm{Si}$ revisan tales suposiciones o ideas sobre la base de las pruebas o del análisis; 5, Si Proponen nuevas observaciones y evaluaciones para esclarecer, modificar y fundamentar las suposiciones e ideas o incluso para generar otras".

análisis de sistemas y resultó dicho enfoque inmerso en el campo de la ingeniería de sistemas. 

tesis.

The qualitative approach in legal research, qualitative research project and thesis seminar

"Sin embargo - concluye el citado autoraunque las aproximaciones cuantitativa y cualitativa comparten esas estrategias generales, cada una tiene sus propias características".

\subsection{Importancia de la investigación jurídica}

Como afirma el profesor Fix- Zamudio (1995, p. 238) en su libro Metodología, Docencia e Investigación Jurídica: "Se advierte en los últimos años una preocupación muy profunda por la adecuación del ordenamiento jurídico al cambio social; especialmente, en Latinoamérica, e inclusive tratadistas de gran calidad científica dudan seriamente de la eficacia del ordenamiento jurídico para resolver los graves problemas derivados del subdesarrollo económico, político y social de nuestros países".

Al respecto de lo mencionado por FixZamudio (1995), mostramos nuestra total conformidad a las dos tesis planteadas, pues actualmente observamos que existe un vivo debate en las diversas casas de estudio, acerca que el Derecho no está cumpliendo de manera adecuada su papel o su rol como agente de cambio y progreso social. Es así, que a causa de esta ineficacia y falta de adecuación del derecho se ha originado una espiral de violencia que afecta el desarrollo científico y cultural de la sociedad.

\section{Investigación jurídico-cualitativa}

\subsection{Concepto}

Con respecto al concepto de investigación cualitativa -Lino Aranzamendi en su libro Investigación Jurídica (2015, p. 148) tiene dicho que- esta investigación "está orientada principalmente hacia la descripción y la comprensión de una situación o fenómeno (caso del Derecho) a diferencia de la cuantitativa que se centra en la cuantificación, predicción y control. En la investigación cualitativa -el precitado autor agrega- que el conocimiento se construye, no se descubre. Con regularidad se tiende a observar, a describir e interpretar lo que se encuentra en los datos (documentales o no), y solo tiene sentido para esa situación o contexto determinado.

Los métodos cualitativos centran su interés en los escenarios naturales y reales en los que los seres humanos interaccionan y se desenvuelven. En estos casos, también prevalecen el análisis y práctica de los valores en tanto estos influyen en el análisis de los problemas y la construcción de las teorías y modelos jurídicos".

\subsection{Rasgos dominantes}

Al respecto, el profesor Aranzamendi (2008, p. 154-155) en su libro Epistemología y la Investigación Cualitativa y Cuantitativa en el Derecho, escribe las siguientes peculiaridades que entraña la investigación cualitativa en los trabajos académicos ligados al mundo del derecho. Son las siguientes:

1. Desde la perspectiva del autor, se considera que la investigación cualitativa es una actividad de carácter sistemático, cuyo fundamento se sustenta en prácticas claramente interpretativas. Asimismo, el aspecto interpretativo ayuda a justificar argumentos a través de un marco teórico y experiencias vividas, respectivamente.

2. Se considera asimismo que en los estudios cualitativos se da mayor y especial atención al contexto o entorno. Por lo demás, el investigador cualitativo desarrolla una sensibilidad hacia situaciones abordadas de manera global u holística.

3. Se señala que la investigación cualitativa, reivindica la reflexividad, lo cual significa que debe prestarse especial atención a la forma que diferentes elementos lingüísticos, sociales, culturales, jurídicos, políticos, y 
teóricos que influyen de forma conjunta en el proceso de desarrollo del conocimiento. La reflexividad supone dirigir la mirada hacia las personas, hechos o fenómenos jurídicos que investiga, el reconocimiento de los supuestos teóricos y también personales (...).

4. La investigación cualitativa tiene un carácter particularista pues centra su estudio en una solo situación, hecho, evento, etc. Además, se estima que la investigación cualitativa resulta adecuada para analizar problemas jurídicos y cuestiones jurisprudenciales.

5. La investigación cualitativa es de índole descriptiva por cuanto identifica los elementos que conforman un caso, hecho o fenómeno de relevancia jurídica, determinando sus conexiones entre sí, permitiendo describir procesos, contextos, instituciones, sistemas y personas (...).

6. Del mismo modo, toda investigación cualitativa "es preponderantemente inductiva en el tipo de razonamiento, casi siempre parte de hechos o fenómenos jurídicos concretos. Lo que significa observar y describir un hecho para posteriormente generar una opción teórica sobre el problema".

7. "En estudios cualitativos raramente es posible formular hipótesis tan exactas que puedan ser rigurosamente verificadas $y$ potencialmente rechazada. Si el investigador ha elegido el método cualitativo y cuando se refiere a la hipótesis, lo que indica es una hipótesis de trabajo, o una suposición que irá gradualmente reforzando o corrigiendo en la medida del avance en el estudio de hecho o caso concreto".

8. Toda investigación cualitativa se sujeta frecuentemente en métodos de recolección de datos sin medición numérica, como las descripciones y las observaciones.
9. Respecto a los métodos, la manera de resolver los problemas que se persiguen, es diseñando métodos flexibles que permitan enfocar y resolver la complejidad del objeto, materia de estudio, en este caso los objetos o hechos no se reducen a variables individuales, sino que se estudia en si su complejidad y totalidad, en su contexto cotidiano e integral.

10. En la investigación cualitativa no se plantea como objetivo probar teorías científicas de validez universal o hipótesis matemáticamente contrastadas.

\section{El enfoque cualitativo y su naturaleza}

El profesor Mejía (2007, p. 146) define a la investigación cualitativa como "el procedimiento metodológico que utiliza palabras, textos, discursos, dibujos, gráficos e imágenes. En este sentido, la investigación cualitativa estudia diferentes objetos para comprender la vida social del sujeto a través de los significados desarrollados por este".

\section{Estrategias del enfoque cualitativo (Diseños básicos)}

\subsection{Teoría fundamentada (Groundad).}

La teoría fundamentada -escribe Katayama (2014, p. 66) - puede ser definida como aquella que se "sustenta en el "interaccionismo simbólico", y "constituye una metodología para desarrollar teorías a partir de datos que son sistemáticamente capturados y analizados". Es un diseño expresa Hernández Sampieri (2018, p. 526)- cuyo propósito es inducir teoría basada con datos empíricos y se aplica a áreas específicas. "El investigador produce una explicación o teoría respecto a un fenómeno, proceso, acción o interacciones que se aplican a un contexto concreto y desde la perspectiva de diversos participantes". Para Nelly Patricia Bautista (2013, p. 123), la teoría fundamentada "es 

tesis.

The qualitative approach in legal research, qualitative research project and thesis seminar

una teoría que contribuye a cerrar la brecha entre la teoría y la investigación empírica y se constituye en una alternativa a las teorías funcionalistas y estructuralistas" (...).

Al igual -prosigue- "que los otros métodos de investigación cualitativa, la teoría fundada contiene muchos puntos de afinidad con otras aproximaciones de investigación cualitativa, por tanto lo importante es encontrar las diferencias o aquellos elementos que la distinguen de los otros métodos. La principal diferencia radica en su objetivo que es la construcción de teoría".

\subsection{Estrategia etnográfica}

El estudio de Hernández Sampieri y Christian Mendoza (2018, p. 537) pretenden "explorar, examinar y entender sistemas sociales: grupos, culturas y sociedades (...), así como producir interpretaciones profundas y significados culturales (...), desde la perspectiva o punto de vista de los participantes nativos". Asimismo, Patton (2015) señala que tales diseños buscan describir, interpretar $\mathrm{y}$ analizar ideas, creencias, significados, conocimientos y prácticas presentes en tales sistemas. Incluso pueden ser muy amplios y abarcar la historia, geografía y los subsistemas socioeconómico, educativo, político y cultural (Rituales, símbolos, funciones sociales, parentesco, migraciones, redes y un sinfín de elementos)"

\subsection{Estrategia de investigación -acción}

Jacqueline Hurtado (2011, p. 105-106) afirma que la investigación interactiva "es aquella, cuyo objetivo consiste en modificar el evento estudiado, generando y aplicando sobre él una intervención especialmente diseñada. En este caso, el investigador pretende sustituir un estado de cosas actual, por otro estado de cosas deseado. La investigación- acción, es una modalidad de investigación interactiva, pero fundamentalmente orientada a las ciencias sociales, y dependiendo de la vertiente puede incorporar la participación de la comunidad estudiada".

Por su parte, Hernández - Sampieri, (2018, p. 552) manifiesta que "la finalidad de la investigación acción es comprender $y$ resolver problemáticas y específicas de una colectividad vinculadas a un ambiente (Grupo, programa, organización o comunidad)". "Asimismo, se centra en aportar información que guíe la toma de decisiones para proyectos, procesos $\mathrm{y}$ reformas estructurales)"

\section{Técnicas del enfoque cualitativo}

\subsection{Observación}

Según Valderrama (2014, p. 272) "la observación es un proceso voluntario $\mathrm{y}$ ordenado que realiza el investigador por una intención, propósito o problema; permite obtener información sobre un caso, hecho o problema para luego describirlo y llevar a cabo el análisis de la información, así como interpretación. Además, la mencionada técnica tiene los siguientes propósitos: a) explorar ambientes, contextos subculturales y la mayoría de los aspectos de la vida social (Grinnell, 1997); b) describir comunidades, contextos o ambientes, así como las actividades que se desarrollan en estos, las personas que participan en tales actividades y los significados de la misma; c) identificar el problema (Daymon, 2010); d) generar hipótesis para futuros estudios".

\subsection{Entrevista cualitativa}

Entendida como una técnica, arte o habilidad práctica, Valderrama (2014, p. 277-278), la define como "herramientas para recolectar datos cualitativos, se emplean cuando el problema de estudio no se puede observar o es muy difícil hacerlo por ética o complejidad (por ejemplo, la investigación de forma de depresión o la violencia en el hogar) y permiten obtener 
información personal detallada. Una desventaja es que proporciona información "permeada" por los puntos de vista del participante".

\section{Campos del enfoque cualitativo}

En sentido amplio y general, los campos del enfoque cualitativo, están relacionados entre sí, porque entrañan ideas o fenómenos complejos subjetivos y comprenden conocimientos de origen social. Así por ejemplo Katayama (2014, pp.43-45 y 47), explica que la investigación cualitativa se orienta a múltiples campos y temáticas, entre las cuales consideramos más importantes las siguientes: "Uno, el conocimiento de fenómenos complejos; Dos, conocimientos orientados al conocimiento de la naturaleza de un grupo social; Tres, dichos campos posibilitan el análisis de fenómenos muy subjetivos; Cuatro, se centran en fenómenos típicamente humanos; Cinco, permiten estudiar los campos en donde se dan las conductas de engaño (...)"

\section{Métodos del enfoque cualitativo}

Los estudiantes, profesores y profesionales emprendedores saben que no sólo existe uno sino varios los "métodos" del "enfoque" cualitativo. Sin embargo, es necesario aclarar previamente que la investigación cualitativa en sì, es un tipo de investigación propiamente dicho. Por consiguiente, cada investigación cualitativa tiene su propia sustancia y sus propio método. Asì también dispone de un conjunto variado de "métodos", tales como el estudio de casos, la observación participante, la entrevista profunda, la investigación participativa, la fenomenología, historia de vida. Asimismo, la teoría fundamentada, la etnometodología, el grupo de discusión o entrevista grupal, el interaccionismo simbólico, la investigación acción participativa, el método etnográfico, el método de la complejidad social, entre otros. (Rivas, 2014, p. 286)
En los últimos años han surgido investigadores que muestran interés por el análisis cualitativo en el campo de las ciencias sociales y humanas. Del mismo modo hay tesistas o maestristas comprensivos que resaltan ideas germinales sobre los métodos del enfoque cualitativo.

Así, de la forma más común ellos generan ideas desde la perspectiva humanista, Weberiana, imaginativa y especulativa. (Graham Gibbs, 2012, p. 20)

\section{El enfoque cualitativo y cuantitativo: Su dicotomía}

Hernández Sampieri y otro, en Metodología de la Investigación (2018, p. 390) señala que La investigación cualitativa "se enfoca en comprender los fenómenos, explorándolos desde la perspectiva de los participantes en su ambiente natural y en relación con el contexto". "Seleccionas el enfoque cualitativo cuando tu propósito es examinar la forma en que ciertos individuos perciben y experimentan fenómenos que los rodean, profundizando en sus puntos de vista, interpretaciones y significados".

Como Rivas (2014, p. 287) en su Diccionario de Investigación Científica Cualitativa y Cuantitativa advierte que los investigadores cuantitativos trabajan con números o guarismos porque la cuantitativa es una investigación orientada a brindar al hombre dominio sobre el universo que se estudia en mediciones y números. El propósito de esta investigación es dar solución a problemas prácticos. En verdad, es un "proceso que gira en torno a datos cuantificables, procedimiento estadístico, construcción de tablas estadísticas (...)”.

"La base filosófica de esta investigación es el empirismo, el racionalismo y el positivismo". El método científico de la investigación cuantitativa es considerado como "método rector" y "universal", 

tesis.

The qualitative approach in legal research, qualitative research project and thesis seminar

absolutamente "único" e "indispensable" para estudiar todo tipo de fenómeno (de la naturaleza como de la sociedad).

Pasemos ahora al análisis de este punto, en el que el mismo Aranzamendi refiere las siguientes ideas, que explica tal dicotomía.

Al respecto, cabe destacar que en el análisis del Derecho como objeto de estudio existen diferentes enfoques epistemológicos, también denominados paradigmas 0 modelos de cientificidad y de investigación (...) Se puede identificar dos enfoques epistemológicos o paradigmas: el normativo, positivista o cuantitativo y el interpretativo, antipositivista, cualitativo o inductivo.

El enfoque cuantitativo como el cualitativo -prosigue- son aplicables en la investigación jurídica. La diferencia se expresa en cuanto al tema de investigación, al método, procedimiento, instrumentos, técnicas y el resultado.

Asimismo, enfatiza el precitado autor que durante décadas ambos enfoques han sido vistos como una dicotomía (en el sentido de "rivalidad"); sin embargo, no debemos verlos como enfoques en competencia, ambos son muy valiosos, ninguno es intrínsecamente mejor que el otro, solo son diferentes aproximaciones. "La investigación cuantitativa nos ofrece la posibilidad de generalizar los resultados más ampliamente, nos otorga control sobre los fenómenos, así como un punto de vista de conteo y más magnitudes de éstos. Nos brinda una gran posibilidad de réplica y un enfoque sobre puntos específicos de tales fenómenos, además que facilita la comparación entre estudios similares". "Por su parte la investigación cualitativa proporciona profundidad a los datos, riqueza interpretativa, contextualización del ambiente o entorno, detalles y experiencias únicas. También aporta un punto de vista "fresco, natural y holístico" de los fenómenos, así como flexibilidad". (Aranzamendi, 2008, pág. 163)

Por último, el mismo autor escribe los siguientes rasgos diferenciadores de dichos enfoques indicando que son los siguientes: a) La cuantitativa se apoya en datos cuantitativos, se tiene como modelo a las ciencias naturales con preponderancia del enfoque inductivo; la cualitativa se apoya en datos cualitativos, en contextos artificiales, rechaza el modelo de las ciencias naturales, privilegia la inducción; b) La cualitativa usa conocimientos propios al Derecho como la hermenéutica; la cuantitativa se recurre a la verificación del fenómeno, uso de gráficos estadísticos, etc.; c) La cualitativa analiza teóricamente los problemas jurídicos; la cuantitativa hace mediciones y cálculos, trabaja con probabilidades; d) La cualitativa es una tarea bastante exigente como análisis teórico; en la cuantitativa bastará la codificación de los elementos frecuentes de un problema (...) (Aranzamendi, 2008)

\section{Categoría político y normativa.}

Cabe resaltar que al ser el Estado, el principal promotor y gestor de la educación en general y de la investigación en particular; debe orientar sus esfuerzos a la implementación de iniciativas de investigación jurídica, con un enfoque innovador y de naciente ingreso como es el cualitativo, a ello sumado la búsqueda del cambio de actitud de los principales actores, entre los cuales tenemos a estudiantes y profesores, hacia un desempeño oportuno en la elaboración y asesoramiento de tesis, guiados bajo este modelo, buscando además una mejora en su implementación pragmática, a través de programas que permitan enfrentar los retos que se tienen actualmente con respecto a este enfoque, en aras de la promoción del mismo.

Así pues, Naupas (2014, p. 463) expone que todo Estado tiene el deber moral de diseñar 
una política científica y tecnológica, acorde con sus necesidades históricas reales, para desarrollar las bases de datos, informaciones y conocimientos fundamentales para luego diseñar políticas acertadas de desarrollo económico-social y cultural. Estas políticas de investigación deben dar respuesta a los principales problemas científico-tecnológicos que afectan a las grandes mayorías y en base a ello orientar la investigación hacia una mejora en la calidad de vida de la humanidad.

Vemos pues que el derecho a la educación en América Latina, Mario Alzamora (1972, p. 29) y la Constitución Política de 1993 (Bernales, 1998) señalan que las constituciones y leyes pertinentes que rigen en los países de América Latina señalan como fines de la educación: la formación de la personalidad humana, la capacitación del hombre para la vida, como individuo y como miembro de la sociedad; la conformación de ciudadanos para la convivencia democrática; la creación, conservación y difusión de la cultura; la incorporación de las grandes mayorías elevando sus niveles de vida y la vigorización del sentimiento nacional.

Así, la situación político-normativa resalta la importancia de la promoción de iniciativas por parte del Estado y los organismos competentes para la creación de estándares sostenibles relacionados a la investigación cualitativa, para lo cual se requiere de canales abiertos que permitan la generación de propuestas encaminadas al fortalecimiento de proyectos investigativos; sobre todo, en materia jurídica cualitativa y que respondan a la insurgente necesidad de una sociedad que se abre al mundo y en la que el mercado fundamentalmente posee una función preponderante en la organización de las relaciones humanas y; en donde, la educación constituye un medio indispensable por la cual las personas pueden asumir conciencia de sus derechos y cumplir un rol en la sociedad.

\section{Categoría social y económica}

Los ingresos, egresos, el status, los estilos de vida, formas, actividades, hábitos, costumbres, etc., son elementos que condicionan que el actuar de las personas sea de tal o cual modo, estas piezas llevándolas al campo de la investigación constituyen una serie de componentes influyentes en la producción científica, sobre todo en los estudiantes de Postgrado de Derecho, quienes en muchas ocasiones se ven desprovistos de una serie de condiciones propicias para el desenvolvimiento efectivo de sus actividades. Las carencias económicas y el posicionamiento en un status social bajo son los principales retos a los que se enfrentan los investigadores, la dificultad en la adquisición de material bibliográfico, medios insuficientes para solventar los gastos derivados del proceso, ambientes carentes de instrumentos mínimos para llevar a cabo el estudio, la necesidad de dedicarse a trabajar para cubrir sus necesidades básicas y; por ende, la investigación reservada en un segundo plano; son solo algunos de los aspectos que llaman la atención y que instan al desarrollo de un marco económico social comprometido con el estudiantado y sobre todo con los investigadores jurídico cualitativos.

Se debe buscar; por tanto, un aumento de la inversión en materia investigativa sobre todo de tipo cualitativo, la creación de un marco más favorable para las empresas y la inversión de las mismas, lograr cambios en los modelos mentales sobre la importancia concedida a las actividades de investigación jurídica e incluirlas dentro de las agendas políticas, a ello sumado el fortalecimiento de una cultura emprendedora y la formación de investigadores cualitativos mediante un aliciente e incentivo de los mismos. 

tesis.

The qualitative approach in legal research, qualitative research project and thesis seminar

\section{Categoría ética.}

Expresa Ñaupas (2014, p. 457) que "el problema moral es decir, indiferencia, desprecio y rechazo de las pautas éticas, que están consagradas por la religión, por la legislación y enseñadas por los padres de familia y las instituciones educativas, al parecer, es un problema humano, casi tan antiguo como el hombre mismo". En tal sentido, al ser la investigación científica una empresa social, es muy probable que al estar contaminada moralmente la sociedad, ello repercuta en los investigadores y científicos.

Y lo que es más, escribe Bernales (1998, p. 217-218) que "el derecho a la educación y a la cultura es inherente a la persona humana. La educación como inherente a la persona humana e instrumento que sirve para el desarrollo integral de la personalidad, es una conquista histórica de la humanidad que corresponde a la sustanciación de la libertad, la igualdad y la racionalidad del ser humano. (...). Es, por tanto, "un proceso que atiende al desarrollo personal del ser humano (...)", lo cual significa que la "educación contribuye a la formación intelectual, profesional y académica de las personas, así como a su realización en todas las dimensiones". "De esta manera, el ser humano tendrá que ser formado como persona, de acuerdo con sus propias características e inclinaciones; pero también tendrá que recibir una educación que lo convierta en un ciudadano responsable y participante".

Bajo esta rúbrica, la situación ética se orienta a ser uno de los pilares que condicionan la elaboración de trabajos de investigación jurídica de corte cualitativo, al desarrollar en estudiantes y docentes sentimientos de trabajo en equipo, cooperación y mutua colaboración; ello acompañado de valores éticos, como la honestidad intelectual, el respeto mutuo, la libertad investigativa, la responsabilidad, entre muchos otros. La dignidad de la persona humana exige, que el hombre en su actuación proceda consciente y libremente, por esta razón el investigador movido por decisiones personales, tomadas por convicción e iniciativa, en actitud de responsabilidad $y$ no en fuerza de imposiciones o presiones se inclinará al estudio cualitativo.

Al ser pues una de las características del investigador cualitativo, su comportamiento razonable y altamente interpretativo guiado por una serie de valores éticos en su actuar, se encontrará unido inexorablemente al sentimiento y la necesidad de mutua colaboración. Al ser los hombres sociables por naturaleza deben vivir los unos con los otros procurando el bien de los demás. Por ello, una convivencia humana bien organizada exige el reconocimiento y el respeto de los derechos y deberes mutuos, mediante el aporte generoso de la colaboración humana, para crear un orden colectivo ciudadano y con ello la generación dé un aporte considerable y oportuno para con la sociedad mediante investigaciones a la luz de este enfoque.

Asimismo, advierte Naupas (2014, p. 466) que "el investigador es un científico, humanista, en el verdadero sentido de la palabra, es decir, un hombre preocupado por la suerte de la humanidad, por sus necesidades, sus vicisitudes, sus angustias, al mismo tiempo que procura su felicidad a nivel de persona y a nivel social. Además, debe ser una persona modesta, honesta, responsable y apasionado amante de la verdad".

Por ello, el investigador debe encontrarse regido principalmente por la honestidad intelectual, como sinónimo de aprecio por la objetividad y el desprecio por el autoengaño, basado en la independencia de juicio, una dosis de coraje intelectual, el amor por la libertad intelectual $y$ fundamentalmente el afianzamiento consiguiente de un sentido de justicia, como 
una disposición a tomar en cuenta los derechos y opiniones del prójimo. Ámbitos de especial consideración dentro de la ética del investigador cualitativo.

\section{PROYECTO DE INVESTIGACIÓN CUALITATIVA}

En los tiempos actuales es una idea generalizada que el estudiante debe obtener el grado de bachiller, el título profesional, grado de maestro y el grado de doctor y para ello tiene que aprender a gestionar proyectos de investigación, la misma que constituye una función esencial $y$ obligatoria porque estamos entrando con una nueva sociedad denominada "era de la información", "sociedad de las nuevas tecnologías". Por lo que se sabe la nueva sociedad abre al estudiante de posgrado toda la posibilidad de crecer creando riqueza y mejorar la calidad de vida y para ello urge generar, emplear y difundir conocimientos científicos, tecnológicos e innovativos.

En efecto, es esta una de las principales razones de hacer con todo el proyecto de investigación. De hecho, habrá que olvidarse de la discusión de si la investigación es un factor importante para crecer económicamente y lograr mejores niveles de vida. Ahora, es urgente en convertirnos como promotores de la gestión y cultura del proyecto de tesis. Al respecto referimos, que son varias las investigaciones sobre enseñanza de los cursos del área metódica que demuestran la necesidad de averiguar los motivos de la baja producción de proyectos en los cursos de Seminario de tesis.

Los profesores deben ser capaces de acomodarnos a los continuos cambios, tanto al contenido de su enseñanza como en la forma de enseñar menos los cursos del área metódica; como el seminario de tesis, por ser ésta disciplina soporte sustantivo en la elaboración del proyecto de tesis.

\section{¿Qué es un proyecto?}

Según el Diccionario de la Lengua Española (2001, p.1852), el proyecto es un " primer esquema o plan de cualquier trabajo que se realiza a veces como prueba antes de darle la forma definitiva".

Por su parte, los metodólogos afirman que el proyecto en sí es un documento donde se especifica qué es lo que se desea investigar y cómo se debe llevar a cabo cada paso para la realización del estudio, por ello es necesario hacer un bosquejo o una pre elaboración $\mathrm{o}$ ante proyecto antes de ejecutar el estudio.

Cabe resaltar lo mencionado por Ramírez Erazo (2010) el proyecto de investigación es una construcción conceptual donde se organiza, planifica, formula y se registra sistemática y racionalmente las estrategias de solución del problema.

\section{Proyecto de investigación cualitativa}

Según Valderrama (2014, p. 311) considera que "se trata de un documento también llamado protocolo (o proyecto) de investigación cualitativa, este contiene la planificación, recolección, análisis de información, etc., y se caracteriza por la descripción y la comprensión de la realidad, dejando de lado la predicción y el control del problema". Se denomina también "protocolo o diseño al plan de trabajo que sirve de instrumento para cumplir el itinerario en el desarrollo de la tesis. Contiene los presupuestos fundamentales estructurados sistemáticamente delimitados: el problema, objeto, variables, marco teórico, la hipótesis y la metodología" (Aranzamendi, 2015, p. 190). Decía Nelly Bautista (2013, p. 139) que el proyecto constituye la "vía de comunicación que el investigador tiene con instancias evaluadoras que examinarán la pertinencia del estudio propuesto". 

tesis.

The qualitative approach in legal research, qualitative research project and thesis seminar

Sobre el proyecto de investigación cualitativa, se ha escrito muy poco; empero, en medio de esta escasez, se distingue el libro Pasos para elaborar Proyectos de Investigación Científica, del profesor Santiago Valderrama, arriba citado. Precisamente, los datos sobre fases o etapas del proyecto de investigación cualitativa, que aparecen más adelante, los hemos tomado de la referida obra.

\section{Fases o etapas}

\subsection{Fase exploratoria y de reflexión}

Como vemos tosa fase exploratoria Consiste en una toma de contacto con los temas de interés del estudio, implica un toma de decisiones sobre aspectos relacionados a la investigación. Incluye una identificación del problema, el planteamiento de las cuestiones de investigación, la revisión documental de la literatura relevante y se debe tener muy en cuenta en esta fase las perspectivas teóricas en que se sustentará el estudio.

\subsection{Fase de Planificación}

Incluye tres etapas: a) La selección del escenario pertinente -subraya Valderrama (2014, p. 315-317)- entraña la idea de "decisión crucial que condiciona todo el desarrollo de la investigación (...)". Sin embargo no todo escenario sirve para la investigación sino que deben observarse reglas, "lo que no quiere decir que cualquier decisión sea válida". "La revisión documental, el contacto con potenciales participantes, la consulta a investigadores expertos y los propios juicios del equipo de trabajo contribuirán a que se tomen decisiones sopesadas y razonadas; b) Toda estrategia de investigación es herramienta de trabajo y es responsabilidad del investigador conocer su variedad y singularidad para el estudio de los fenómenos sociales; y c) Redefinición del problema, estableciendo nuevas categorías y mejorando el proceso investigativo concluye el precitado autor.

\subsection{Fase de entrada en el escenario}

Comprende como primer paso negociar el acceso, lo cual requiere tiempo, persuasión, formulación de convenios, tacto y sensibilidad hacia los ritmos y normas de la institución y personas implicadas, viene luego la selección de los participantes, esta constituye la parte fundamental del trabajo cualitativo e implica el empleo de una serie de estrategias por parte del investigador, el cual debe ser sensible y abierto líder y supervisor, debe pues conocer el tipo de muestreo intencional pertinente, estos pueden ser de los siguientes tipos: a) De variación máxima, b) Por tipos de casos, c) Comprensivo d) En red.

\subsection{Fase de recojo $y$ análisis de la información}

Plantearse la cuestión de recojo y análisis de la información en el ambiente natural de los participantes, implica un serio esfuerzo porque el fruto de dicha búsqueda o recojo o hallazgo "datos ricos y profundos, así como el interés en la descripción de los hechos" respectivos (Solís, 2008, p. 94).

Esta manera de entender el recojo de datos como búsqueda y hallazgo, nos lleva a pensar con Hernández Sampieri (2018), que tal recojo se apoya en las anotaciones tomadas en libretas o cuadernos de bitácora, en la observación, entrevista, etc. Para él la recogida es el "acopio de datos narrativos en los ambientes naturales y cotidianos de los participantes o unidades de muestreo" (2018, p. 443). Asimismo, él considera que "el instrumento del recojo de los datos cualitativos, es el investigador auxiliándose de diversas herramientas como las entrevistas, la observación y las sesiones grupales" (Hernández, 2018, p. 443) . Por último, el citado autor afirma que se recolectan los datos con la finalidad de ser 
analizados y comprendidos, a la vez que tratan de responder a las interrogantes de la investigación y de esa forma generar nuevos conocimientos.

\subsection{Fase de retirada del escenario}

En el punto anterior se trató del recojo y análisis de la información ahora vamos a tratar la retirada de escenario o contexto. ¿Qué es la retirada del escenario?. Ésta, en cierto modo, es una cuestión última relevante de las fases o etapas de la investigación cualitativa. Como su nombre lo indica retirada es el retiro o retorno, distancia o alejamiento del lugar donde pernoctan los participantes del trabajo de investigación.

Sin duda es este el momento en que nos apartamos los miembros del Grupo de Investigación (GI); es decir, nos separamos de sitio o lugar natura donde ellos habitan cotidianamente. Un momento en que nos alejamos por haber llegado al punto de saturación de la recolección de la información. Se pone fin pues porque se han obtenido las ideas, creencias, imágenes mentales, vivencias y experiencias que han sido vertidas oportunamente en el lenguaje de las unidades participantes. (Hernández Sampieri, 2018 Valderrama, 2014).

\subsection{Elaboración del informe}

Para entender lo que es la elaboración del informe final, es indispensable señalar primero que es "necesario redactar dicho informe para comunicar los resultados hallados a la entidad o personas que apoyaron o financiaron el trabajo, y también para conocimiento de la comunidad científica, entre otros. Al respecto, el estudio más riguroso así como los resultados más importantes, tendrán un valor mínimo o escaso sino son divulgados, ya que así no se dan a conocer es imposible que puedan servir para algo" (Solís, 2008, p. 259).
Además resulta importante establecer la "estructura del contexto académico y que es como sigue: portada, índice, resumen, términos clave, cuerpo del documento o trabajo (introducción, revisión de la literatura o marco teórico, métodos, análisis de resultados, discusión, referencias o bibliografía y apéndice)". Del mismo modo, "en contextos no académicos la estructura básica es: Portada, índices, resumen ejecutivo, método (abreviado), resultados, conclusiones y apéndices" (Hernández, et al, 2018, p.571).

Es muy interesante lo que expresa Gibbs (2012) haciendo referencia a la bitácora del día o diario de investigación en el que los investigadores toman nota de sus diálogos o tertulias con las unidades participantes en la ejecución del proyecto. Según él la manera como los investigadores hacen trabajo de campo haciendo uso no solo de libretas o cuadernos, sino también efectuando simultáneamente notas o apuntes de campo, en el preciso momento de su actuación en el sitio o lugar de la investigación. Por último, el citado autor, revela que no es solo la bitácora del día sino también notas de campo, que importan ya una faena, plenamente imaginativa y creadora (no solo redactan ideas y hechos, sino que también los analizan e interpretan).

\section{SEMINARIO DE TESIS}

\section{Idea General}

En el Diccionario de la Lengua Castellano (Bouret), el seminario es el criadero o plantel destinado para que crezcan las plantas en cierto tiempo, hasta la época de su trasplantación. Local exclusivamente destinado para la buena educación de los niños y jóvenes. Seminario conciliar, es aquel especialmente destinado para la educación religiosa, cuyos jóvenes salen regularmente a ser curas de almas, después de haber cursado gramática latina, algo de matemáticas, filosofía y teología. 

tesis.

The qualitative approach in legal research, qualitative research project and thesis seminar

Seminarista es el alumno que practica la técnica del debate y discusión sobre temas de interés para los seminaristas.

\section{Lo que es el Seminario de Tesis}

El seminario de tesis, conforme a la concepción moderna, es una disciplina que consiste en el estudio y análisis de los diferentes pasos de la formulación de un proyecto, siendo el resultado esperado la construcción completa del proyecto de investigación, obtenido como un bello producto, original y personal de cada participante. Nosotros entendemos al seminario de tesis como una disciplina que facilita al futuro magister los conocimientos y ejercicios propios de la formulación del proyecto, teniendo como base el silabo, que sustenta las principales unidades temáticas del curso. Dichas unidades son abordadas, una a una en clase, en forma sistemática y ordenada, en el contexto de un trabajo de aula, con horas de clase en que se combina la clase magistral, con el debate y discusión de proyectos elaborados por cada uno de los participantes del seminario.

La organización fundamental del seminario gira en torno al tema, el proyecto, la coordinación, discusión y debate que realizan los participantes, bajo la guía y conducción del profesor durante la sesión del seminario.

El contenido temático entraña la enseñanza para que los estudiantes aprendan los pasos de la investigación jurídica formal y los pasos de la investigación jurídica cualitativa y cuantitativa, así como enseñar a aprender los pasos de la investigación teórica o dogmática

Es más, se trata de proporcionar al maestrista la orientación e información necesaria que le permita identificar la naturaleza, concepto, estructura, formulación y aprobación de cada uno de los enfoques del proyecto que presenta el alumno; asimismo, dar una visión panorámica de la forma como se elabora paso a paso, un modelo de investigación jurídica cualitativa $y$ un modelo de investigación jurídica cuantitativa.

Respecto al procedimiento de las sesiones del seminario los participantes son entrenados individualmente en la formulación del proyecto con la ayuda, guía y conducción del profesor. Se aplica una metodología dinámica con la activa intervención del participante, conjugando la participación docente con el seminario y el trabajo grupal intenso hasta la culminación del proyecto de tesis de cada uno de los educandos.

\section{Planeamiento de las sesiones del seminario}

En todo momento, el planeamiento didáctico, se orienta al reforzamiento de una educación jurídica, rica en valores, principios, decencia y honestidad intelectual y profesional. La intención de apuntar hacia una formación integral, con presencia de actitudes, valores, intereses, y expectativas, con el objeto de formar maestros investigadores, capaces no solo de dominar nuestra propia realidad local, regional y nacional, sino también de formar una conciencia de transformación y de aportar eficazmente con esa conciencia al proceso de cambio que reclama la comunidad a su universidad.

\section{3. ¿Qué se hace y cómo se hace para elaborar el planeamiento de las sesiones del seminario?}

Para elaborar dicho planeamiento es necesario la consulta de los especialistas, quienes responden que el planeamiento $o$ preparación de la sesión de clase consiste en que el profesor, antes de ir al aula debe conocer con precisión el ámbito y marco general de los temas de la sesión del seminario y prever el procedimiento a utilizar para desarrollar dicho tema, con la 
activa participación del estudiante de postgrado; por esta razón, y para comprender la forma en que se desarrolla el seminario de tesis, debemos brevemente detenernos en los pasos del planeamiento didáctico aplicado en cada una de las acciones del trabajo.

\section{Pasos del planeamiento didáctico}

Desde luego, es esta una tarea prioritaria, reinante en todas las sesiones de trabajo, haciéndose efectiva en base a las necesidades, posibilidades e intereses del educando.

Consideramos, que otro paso inicial de las primeras sesiones del seminario es el desarrollo de un esquema orientativo para la formación de la bibliografía de los temas propuestos por los alumnos. Con ello, no solo se persigue identificar lo que ya se conoce y lo que se desea conocer sobre los temas de investigación, sino también iniciar la búsqueda de las bibliotecas públicas $\mathrm{y}$ privadas para la consulta de las fuentes necesarias: revistas especializadas, artículos académicos, ensayos, textos, diccionarios, enciclopedias jurídicas, etc., que guardan relación con el tema de la tesis.

Un tercer paso que se da es el de examinar los puntos propuesto en el proyecto de tesis. Lo cual comprende la definición del trabajo. Vale decir elaborar el enunciado del tema, con exposición de los motivos de su elección y referir lo que se propone a

\footnotetext{
${ }^{7}$ Con el artículo 43 de la Nueva Ley Universitaria $\mathrm{N}^{\circ}$ 30220 los estudios de posgrado conducen a Diplomados, Maestrías y Doctorados. Los diplomados son estudios de perfeccionamiento profesional en áreas específicas y se debe completar un mínimo de veinticuatro (24) créditos. Respecto a las maestrías tenemos las de especialización que son estudios de profundización profesional; $\mathrm{y}$, maestrías de investigación o académicas que son estudios de carácter académico basados en la investigación. Se debe completar un mínimo de cuarenta y ocho (48) créditos y el dominio de un idioma extranjero.
}

investigar. Asimismo, el alumno con ayuda de los participantes y del profesor, debe enumerar los aspectos que presenta el tema elegido, con indicación de aquellos en los cuales va a centrar la investigación. Además, este tercer paso, comprende la fijación de objetivos científicos que se pretende lograr $\mathrm{y}$ el enunciado de las interrogantes que plantea el problema de investigación elegido, respectivamente.

Se conoce en el Perú con el nombre de Unidad de Posgrado de Derecho (UPG). Durante sus años de vida institucional y particularmente en los últimos dos decenios, nuestra Unidad de Posgrado muestra avances significativos ${ }^{7}$. Las primeras vivencias se dan en los alumnos, desde el primer día de clase, con la participación en las sesiones del seminario de cada asignatura, a cargo de destacados educadores. Ellos y ellas suscitan interés y explican los asuntos de clase, en el contexto de un alturado debate y discusión, con sugerentes explicaciones que dan lugar al surgimiento de un impacto que siente el alumno, desde el comienzo hasta el fin de clase $^{8}$.

Más allá de las teorías, las vivencias de los primeros días de formación el alumno infiere que lo que persigue el currículo es no solo brindar conocimientos, sino que también se preocupa del desarrollo de la persona a través de condiciones propicias y oportunidades que se brinda al alumno para lograr habilidades y competencias, así

\footnotetext{
Luego el doctorado que son estudios de carácter académico basados en la investigación. Tienen por propósito desarrollar el conocimiento al más alto nivel. Se debe completar un mínimo de sesenta y cuatro (64) créditos, el dominio de dos (2) idiomas extranjeros, uno de los cuales puede ser sustituido por una lengua nativa.

8 El educador no solo forma profesionales sino principalmente prepara investigadores. Es mediador, cuya función principal es potenciar las capacidades de los estudiantes (...) Ver PEI20122021, página 31 .
} 

tesis.

The qualitative approach in legal research, qualitative research project and thesis seminar

como captar y realizar una amplia gama de valores, además del valor de la verdad.

El educador en el Posgrado ${ }^{9}$ tiene una visionaria concepción educativa porque así como la Universidad, ella también tiene un porvenir lleno de urgencias y desafíos que es necesario afrontar precisamente con esta visionaria concepción educativa. Desde luego, debe responder a este reto con una actitud prospectiva, digna de los nuevos tiempos, con una escuela activa en la que el alumno debe ser educado para la acción, para que desarrolle sus potencialidades al máximo. No hay mejor investigación ${ }^{10}$ que cuando el alumno hace y actúa; es decir, es protagonista de algo y decide hacer el proyecto de tesis y ejecutarlo, utilizando el aplicativo cualitativo o cuantitativo. Lo importante es que el aplicativo cualitativo crezca elevando su actual condición inicial o germinal en el que se encuentra, según los resultados obtenidos en este trabajo.

\section{CONCLUSIONES}

El estado de los conceptos o categorías, en que se afianza el estudio, como lo cognitivo, metódico, político, etc. es de índole inicial, con una clara tendencia hacia un crecimiento institucional.

Las fuentes consultadas resaltan que el enfoque cualitativo es novedoso, complementándose con el enfoque cuantitativo y lo asocian significativamente con el sentido interpretativo; asimismo, apuntalan que es poca la información bibliográfica y de campo que se tiene sobre este enfoque y que es conveniente potenciar dicha información.

\footnotetext{
${ }^{9}$ El art. 112 del Estatuto de la UNMSM., establece que los estudios de postgrado conducen a la obtención de las diplomaturas, los títulos de segunda especialidad o especialista y los grados académicos de maestría y doctorado. Estos se desarrollan en la Unidad de Postgrado del Vicedecanato de Investigación y Postgrado de la Facultad.
}

Se aprecia que el estado de dicho enfoque cualitativo arriba indicado se afianza en las siguientes dimensiones situacionales: cognitivo-metodológica, políticonormativa, socio-económica y ética.

En la literatura materia de consulta se denota una marcada motivación pedagógica para emprender la elaboración y ejecución de proyectos de investigación, con enfoque cualitativo en su afán de optar el grado académico de magister en la Unidad de Posgrado de Derecho.

Según las referencias bibliográficas el enfoque cualitativo es un aborde interno, subjetivo e interpretativo que permite hacer cuestionamientos sobre la realidad jurídicosocial; asimismo, destacan que con este enfoque se mejoran las propuestas de estudio; y que en su elaboración mucho influyen la formación profesional, la capacitación y la experiencia profesional.

Las normas legales así como los Estatutos de las Unidades de Posgrado de Derecho alientan a la investigación; sin embargo, refieren que dicha normativa es perfectible para lograr un crecimiento y desarrollo.

Las unidades y escuelas de Posgrado de Derecho del país participan en el proceso de internacionalización a favor de una universidad de calidad en la formación universitaria y en la investigación en el posgrado.

\section{RECOMENDACIONES}


Organizar eventos y otros certámenes sobre el enfoque cualitativo de la investigación jurídica.

Difundir mediante campañas los alcances y proyecciones de la investigación jurídica con enfoque cualitativo.

Promover la capacitación docente en materia de aplicaciones del enfoque cualitativo en las distintas áreas de la investigación jurídica.

Organizar jornadas de investigación cualitativa anualmente.

Impulsar el reequipamiento permanente de la biblioteca y hemeroteca de la Unidad de Posgrado sobre todo con materiales sobre investigación jurídica cualitativa.

\section{FUENTES DE INFORMACIÓN}

\section{Fuentes bibliográficas}

Alzamora Valdez. M. (1972) El Derecho a la Educación en América Latina. Lima, Perú: Lib. Studium S.A.

Amat, C. y León Ch. (2015) Nueve ensayos para discutir y decidir El Perú Nuestro de Cada Día. $2^{\circ}$ Edición.Lima Perú: Universidad del Pacífico.

Aranzamendi, L. (2015) Investigación Jurídica. $2^{\mathrm{a}}$ edición. Lima, Perú: Ed. Jurídica Grijley.

Aranzamendi, L. (2008) Epistemología y la Investigación Cualitativa y Cuantitativa en el Derecho. $1^{\mathrm{a}}$ Edición. Lima, Perú: Ed. ADRUS.

Aristóteles. (1947). Obras Completas. Tomo II. Traducción de Patricio de Azcárate. Buenos Aires, Argentina: Ediciones Anaconda.
Bautista, N. (2013) Proceso de la Investigación Cualitativa (Epistemología, metodología y aplicaciones). Bogotá, Colombia: Ed. Manual Moderno.

Bernal, C. y et al (2014). Fundamentos de Investigación. $1^{\circ}$ Edición. México: Pearson Educación

Bernales E. (1998) (en colaboración de Alberto Otárola). La Constitución de 1993. Lima, Perú: Ediciones Constitución y Sociedad..

Bunge, M. (2000) Epistemología. Curso de actualización. $2^{a}$ edición. Ed. siglo veintiuno editores S.A. Barcelona. España. 2000 .

Dynnik. M. A. y otros. (1962) Historia de la Filosofía (De la antigüedad a comienzos del Siglo XIX). México: Ed. Grijalbo S. A. México.

Facultad de Ciencias Sociales UNMSM. (2017) V Congreso Internacional PreALAS Perú 2017 Crisis de Civilización: Ser, Saber y Poder en América Latina y el Caribe. Primera Edición. Impresiones y ediciones Arteta E.I.R.L. Lima-Perú.

Fix-Zamudio, H. (1995). Metodología, docencia e investigación jurídica. $4^{\mathrm{a}}$ edición. México: Ed. Porrúa S.A.

Gibbs, G. (2012). El análisis de datos cualitativos en Investigación Cualitativa: Ediciones Morata.

Hernández, R. et al. (2014) Metodología de la Investigación. $6^{\mathrm{a}}$ edición. México: Ed. Mc Graw Hill Education.

Hernández Sampieri, R. y Mendoza Torres P. (2018). Metodología de la Investigación. $1^{\mathrm{a}}$ edición. México: Ed. Mc Graw Hill Education. 

tesis.

The qualitative approach in legal research, qualitative research project and thesis seminar

Hurtado de Barrera, J. (2011) El proyecto de investigación holística. $2^{\mathrm{a}}$ edición. Bogotá, Colombia: Ed. Corporativa Magisterio.

Katayama Omura, R. (2014) Introducción a la investigación cualitativa: Fundamentos, métodos, estrategias y técnicas. Primera Edición. Lima, Perú: Fondo ed. de la UIGV.

Mejía Navarrete J. (2009) Investigación Cualitativa: Nuevos conceptos y campos de desarrollo (Comp). Lima, Perú: Unidad de Posgrado de la Facultad de Educación de la UNMSM.

Naupas Paitán, H. y otros. (2014) Metodología de la Investigación Cuantitativa-cualitativa y redacción de tesis. $4^{\mathrm{a}}$ edición. Bogotá, Colombia: Ediciones de la U.

Real Academia Española. (2001) Diccionario de la Lengua Española. I Tomo y II. 22 a edición. Editorial Espasa Calpe S.A. Buenos Aires. Argentina.

Rivas Ñañez, F. (2014) Diccionario de investigación científica cualitativa y cuantitativa. $1^{\circ}$ Edición. Editado Por el Consejo Nacional de Ciencia y Tecnología e Innovación Tecnológic Concytec. Lima,Perú.

Sainz de Robles, F. (1977) Ensayo de un diccionario español de sinónimos y antónimos. $1^{\text {a }}$ edición. Editorial Aguilar. Madrid, España.

Solís Espinoza, A. (2008) Metodología de la investigación jurídico social. $1^{\mathrm{a}}$ edición. Lima, Perú: Ed. FECAT E.I.R.L.

Unidad de Posgrado de Derecho y Ciencia Politica UNMSM. (2006) Jornada de evaluación curricular. $1^{\mathrm{a}}$ edición. Lima, Perú: Talleres gráficos del centro de producción ed. e imprenta de la Universidad Nacional Mayor de San Marcos.
Valderrama Mendoza, S. (2014) Pasos para elaborar proyectos de investigación científica. $2^{\mathrm{a}}$ reimpresión. Lima, Perú: Ed. San Marcos.

Vicerrectorado Acadèmico UNMSM. (2016) Estatuto Universitario (de conformidad con la Ley N. ${ }^{\circ}$ 30220). Editorial e imprenta de la Universidad Nacional Mayor de san Marcos. Lima. Perú.

Vicerrectorado de Investigaciòn UNMSM. (2013) Reglamento de gestión de actividades de investigación. Talleres gráficos del centro de producción, editorial e imprenta de la universidad Nacional Mayor de San Marcos( Cepredim). 